\title{
Alterstice
}

Revue internationale de la recherche interculturelle

International Journal of Intercultural Research

Revista International de la Investigacion Intercultural

\section{Identité nationale ou identité régionale ? Une étude de cas à travers le conflit martiniquais de février 2009}

\section{Yasmine Diana Misantrope et Anne-Marie Costalat-Founeau}

Volume 4, numéro 1, 2014

URI : https://id.erudit.org/iderudit/1077481ar

DOI : https://doi.org/10.7202/1077481ar

Aller au sommaire du numéro

Éditeur(s)

Alterstice

ISSN

1923-919X (numérique)

Découvrir la revue

Citer cet article

Misantrope, Y. D. \& Costalat-Founeau, A.-M. (2014). Identité nationale ou identité régionale ? Une étude de cas à travers le conflit martiniquais de février 2009. Alterstice, 4(1), 45-59. https://doi.org/10.7202/1077481ar
Résumé de l'article

Cette étude nous permet d'observer la dynamique qui se joue dans la construction de l'identité martiniquaise à travers le conflit de février 2009. C'était une situation à la fois originale et complexe et c'est avec une recherche longitudinale effectuée en situation naturelle que l'on tente de révéler les mécanismes identitaires. Nous avons choisi de l'appréhender grâce au modèle de l'ego-écologie de Zavalloni et Louis-Guérin, appliqués à plusieurs cas, et nous présentons ici nos résultats pour un sujet emblématique des discours étudiés. Nous avons ainsi pu observer l'utilisation de stéréotypes et un rejet du groupe des Français, ainsi que la mise en place de stratégies de reconnaissance. La quête de reconnaissance s'est révélée à la fois génératrice de conflit et régulatrice de l’identité. 


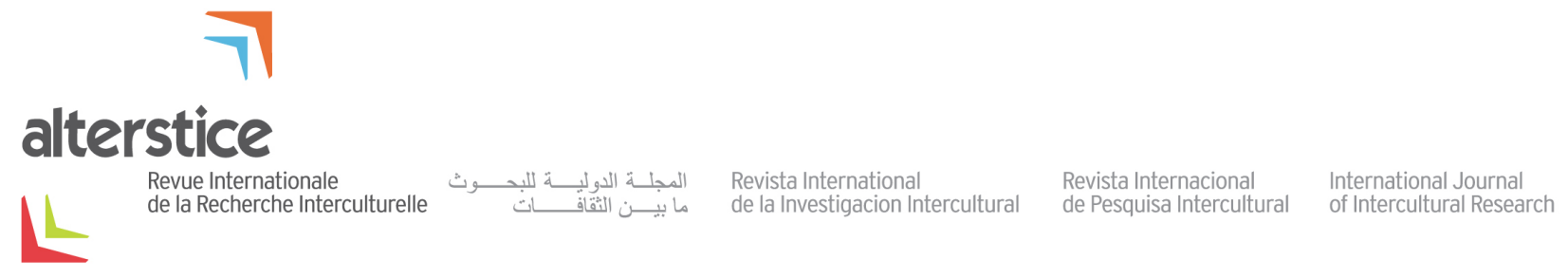

ARTICLE HORS THÈME

\section{Identité nationale ou identité régionale? Une étude de cas à travers le conflit martiniquais de février 2009}

Yasmine Diana Misantrope ${ }^{1,2}$ et Anne-Marie Costalat-Founeau ${ }^{1}$

\section{Résumé}

Cette étude nous permet d'observer la dynamique qui se joue dans la construction de l'identité martiniquaise à travers le conflit de février 2009. C'était une situation à la fois originale et complexe et c'est avec une recherche longitudinale effectuée en situation naturelle que l'on tente de révéler les mécanismes identitaires. Nous avons choisi de l'appréhender grâce au modèle de l'ego-écologie de Zavalloni et Louis-Guérin, appliqués à plusieurs cas, et nous présentons ici nos résultats pour un sujet emblématique des discours étudiés. Nous avons ainsi pu observer l'utilisation de stéréotypes et un rejet du groupe des Français, ainsi que la mise en place de stratégies de reconnaissance. La quête de reconnaissance s'est révélée à la fois génératrice de conflit et régulatrice de l’identité.

\section{Rattachement des auteures}

${ }^{1}$ Laboratoire Epsylon EA4556 et Université Paul-Valéry Montpellier 3, Montpellier, France

${ }^{2}$ Institut universitaire de technologie Montpellier-Sète, France

\section{Correspondance}

yasminemisantrope@gmail.com

\section{Mots clés}

identité; conflit; reconnaissance

\section{Pour citer cet article}

Misantrope, Y. et Costalat-Founeau, A.-M. (2014). Identité nationale ou identité régionale? Une étude de cas à travers le conflit martiniquais de février 2009. Alterstice, 4(1), 45-60. 


\section{Introduction}

En février 2009, la Martinique a traversé un conflit social comme elle n'en avait pas connu depuis les années 1970, avec la grève de l'Union des travailleurs agricole, qui à l'époque a été rejointe par la jeunesse étudiante et lycéenne (Desplan, 2010). Nous avons eu l'occasion de vivre l'impact de ce conflit, dont le point de départ était la vie chère. C'est une situation à la fois originale et complexe, et c'est par une recherche longitudinale effectuée en situation naturelle que l'on a voulu saisir à cette occasion les changements éventuels dans les mécanismes identitaires des Martiniquais, que nous étions justement en train d'étudier (Misantrope, 2011).

L'objectif de notre recherche était d'explorer l'identité psychosociale des Martiniquais, identité contrariée par les stigmates d'un passé colonial encore présent. Nous pensons que c'est le manque de reconnaissance qui a constitué le sous-bassement du conflit social qui a eu lieu à ce moment-là. Nous avons décidé d'appréhender l'identité dans une approche qui intègre à la fois la quête de reconnaissance (Honneth, 2000) et le rôle du conflit en tant que phénomène stable dans l'histoire de la Martinique (Desplan, 2010). En effet, les Martiniquais ont la sensation que leurs difficultés, au niveau tant social qu'économique, sont liées à leur statut de dominés et aux particularités de leur histoire, de leur culture et d'une économie peu développée. "Le signe du pouvoir, c'est le sentiment d'être traité en objet : objet de procédure, de décision, de mesure, de règlement, parfois [...] en objet de sollicitude ou de concertation, mais en objet tout de même » (De Gaulejac et Taboada-Leonetti, 1994).

Nous avons travaillé sous l'angle de l'approche ego-écologique ainsi que de l'Investigateur multistades de l'identité sociale (IMIS) développés par Zavalloni et Louis-Guérin (1984), car cela paraissait adapté au recueil des éléments nécessaires à notre analyse. Nous présentons ici l'un des cinq cas étudiés avant et après le conflit. Grâce à une discussion conclusive, nous dégageons, pour ce cas, les processus susceptibles d'être à l'origine des transformations générées par le conflit. Nous espérons que notre analyse qualitative rendra compte de l'originalité de l'identité martiniquaise.

\section{Le contexte martiniquais : une identité enracinée dans une histoire complexe}

À cause de son passé colonial, la France a toujours eu une place de dominant dans ses rapports avec la Martinique. La départementalisation, obtenue le 9 mars 1946 par le député-maire Aimé Césaire, a permis aux Martiniquais d'être reconnus comme des citoyens français à part entière. Cependant, au contraire des autres anciennes colonies françaises, la Martinique, par la présence des békés, descendants des anciens colons, conserve une trace vivace de cette colonisation. Ainsi, en 2008, alors que l'île compte environ 400000 habitants dont les békés forment simplement $1 \%$, ces derniers posséderaient plus de $50 \%$ de ses richesses (Gurrey et Hopkin, 2009). Ils constituent " une minorité économiquement dominante [...] donc par leur rôle et leur pouvoir une véritable majorité sociologique » (Benoist, 1972, p. 83). De ce rapport dominé-dominant hérité d'un passé colonial où la culture " nègre » était niée, est né un besoin de reconnaissance qui semble se manifester de manière structurelle dans le conflit, qui est "l'explosion d'une identité longtemps contrariée, parfois niée, et finalement libérée et qui, se libérant, s'affirme en vue d'une reconnaissance » (Césaire, 2008, p. 89).

\section{Culture, construction identitaire et acculturation}

La Martinique n'est pas née "du fait du prince ", elle a ses propres racines culturelles et géographiques, influencées par son positionnement périphérique. L'identité culturelle n'est pas une affaire d'essence : elle relève plutôt de la dynamique de la construction sociale (Kozakaï, 2000). La culture est le facteur essentiel qui donne forme à l'esprit de celui qui vit en son sein et est davantage un produit de l'histoire qu'un produit de la nature (Bruner, 1991). Elle est « l'ensemble [...] des significations acquises les plus persistantes et les plus partagées que les membres d'un groupe, de par leur affiliation à ce groupe, sont amenés à distribuer de façon prévalente sur les stimuli provenant de leur environnement et d'eux-mêmes, induisant vis-à-vis de ces stimuli des attitudes, des représentations et des comportements communs valorisés dont ils tendent à assurer la reproduction par des voies non génétiques » (Camilleri et Cohen-Emerique, 1989, p. 27). 
La culture d'un groupe, pour qu'elle continue à exister, doit être valorisée par ce groupe, doit avoir une certaine visibilité et doit être reconnue par l'Autre (alter), notamment quand une relation est établie sur le mode dominédominant. Dans toute relation, le Soi se retrouve face à un alter, avec lequel il peut être sur un pied d'égalité ou dans un rapport hiérarchique. Selon Mead (1963), le Soi correspond à l'ensemble des images que les autres nous renvoient de nous-mêmes et que l'on intériorise. Or, "[l]e problème de la construction des identités à la Martinique s'avère redoutable en raison d'un manque constitutif. L'altérité du Maître, faute d'avoir été combattue et vaincue, fut intériorisée. Elle ne se positionne donc plus en face du sujet mais en lui, puisque non seulement il a accepté sa situation actuelle mais, de surcroît, il l’a désiré » (Affergan, 2006, p. 13).

Redfield, Linton et Herskovits (1936) définissent l'acculturation comme étant " un ensemble de phénomènes résultant d'un contact continu et direct entre groupes d'individus appartenant à différentes cultures et aboutissant à des transformations affectant les modèles culturels originaux de l'un ou des deux groupes " (p. 149). II est important de noter que cette définition de l'acculturation implique, en principe, des changements mutuels entre les groupes en présence mais, en pratique, il arrive que le groupe dominant jouisse d'une influence plus forte que le groupe non dominant (Berry, 1977). "L'acculturation inscrite au cœur du processus de métissage assimilateur antillais autorise une forme d'identification à l'altérité sans laquelle le Martiniquais n'aurait jamais pu accéder au statut juridique de citoyen français. Les deux mécanismes cohabitent sans former une unité. Nous serions donc en présence d'un modèle de métissage où certains fils ne se mêlent pas à d'autres » (Affergan, 2006, p. 28).

\section{Entre culture martiniquaise et nationalité française}

"La citoyenneté démocratique moderne, organisée dans le cadre de la nation, a été l'instrument prééminent de l'unification " (Doytcheva, 2005, p. 72). La nationalité est en effet la condition d'existence de la citoyenneté, puisque seul le national est citoyen (article 3 de la Constitution). Deux conceptions de la nation s'opposent traditionnellement : I'une, ethniciste, l'autre, se voulant civique. Dans la première, le lien entre l'individu, sa terre et ses morts interdit toute autonomie. La nation désigne alors l'organisation naturelle d'une communauté d'origine, basée sur l'ethnie et la langue. Dans une conception plus civique, la nation est définie comme une grande solidarité constituée par le sentiment des sacrifices que l'on a faits et de ceux qu'on est disposé à faire. La nation procède du désir de vivre en commun et s'inscrit dans le prolongement de la philosophie du contrat de Renan parue en 1882.

Thiesse (1999) offre une conception qui diffère des précédentes. Elle considère que les nations et les identités nationales ne sont pas inhérentes à la nature humaine, mais qu'elles ont une histoire. La nation est une création en trois parties: l'identification des ancêtres, le folklore et la culture de masse. Dans cette conception " constructiviste ", il paraît peu probable que les Martiniquais s'identifient aux mêmes ancêtres ou partagent le même folklore que les Français. "L'inculcation de cette identité nationale, si elle a parfois recours à la coercition, notamment en France, [...] n'est réellement efficace et mobilisatrice que si elle est acceptée et intériorisée par une grande part de la population » (Taboada-Leonetti, 2000, paragraphe 6).

L'identité ethnoculturelle "n'est rien d'autre qu'un sentiment d'appartenance ou une identification à une collectivité » (Sméralda, 2008, p. 11), elle est constituée des représentations que les individus se font de la réalité sociale et de ses divisions. Or les Martiniquais se distinguent des Français, qu'ils assimilent aux békés, cette différenciation culturelle demeurant un vestige de l'ère coloniale. L'enclave békée et la communauté francohexagonale sont exclues de la problématique du métissage que propagent la dynamique fusionnelle dominante et les pressions sociales qui assortissent l'intégrationnisme d'exigences relationnelles croisées (Sméralda, 2008). La complexité qui entre en jeu lorsque l'on s'intéresse aux domaines de la culture, de la nationalité et du partage du territoire avec les békés, sur le terrain martiniquais, offre la particularité de conjuguer une proximité juridique à un éloignement symbolique (Affergan, 2006).

\section{Cadre théorique}

Nous définissons dans cette section les différents concepts utiles à notre démonstration, qui vise à prouver que c'est le manque de reconnaissance qui a constitué le sous-bassement du conflit social qui a secoué la Martinique en 2009. Nous précisons ici les liens entre reconnaissance, identité et conflit et nous présentons la démarche egoécologique qui a guidée notre recherche. 


\section{Identité et reconnaissance}

Selon Honneth (2000), la reconnaissance morale se subdivise en trois sphères intimement liées. La première, la sphère de l'amour, suppose que la relation de reconnaissance est liée à l'existence d'autres personnes avec lesquelles la personne fait l'expérience d'une reconnaissance affective et peut développer à l'égard de "soimême " une attitude de confiance se traduisant par une sécurité émotionnelle dans l'expression de ses besoins. La deuxième, la sphère du droit, suppose qu'une personne puisse se sentir porteuse des mêmes droits qu'autrui et développer ainsi un sentiment de respect de soi. Ici, la relation de reconnaissance se fonde sur des droits et des devoirs égaux. Enfin, la troisième sphère correspond au modèle de la contribution à la société, ce qui suppose que l'apport des sujets à la collectivité, dont les particularités individuelles se sont construites à travers une histoire de vie singulière, soit considéré sans discrimination et qu'ils puissent ainsi développer un sentiment d'estime de soi. La quête de reconnaissance peut revêtir des formes variées qui se déclinent en série de " besoins identitaires " (Marc, 2005), qui seront d'autant plus pressants que les individus se sentent en position d'insécurité, d'infériorité et d'exclusion. La reconnaissance implique un rapport à autrui, à un autre avec lequel nous entretenons des relations d'échange. Or, si ces échanges s'effectuent de manière déséquilibrée, le conflit apparaîtra. En effet, le déséquilibre intervient lorsqu'un rapport de position ne permet plus à chacun de conserver à ses propres yeux et de défendre devant les autres une image valorisante de lui-même. "Le sentiment d'être passé à travers les mailles de la reconnaissance sociale représente une source de motivation hautement ambivalente, à la fois de la révolte sociale et de la résistance; cette motivation manque de toute orientation normative permettant de montrer les voies d'un combat, d'une lutte contre l'expérience du mépris et de l'humiliation » (Honneth, 2008, p. 201).

\section{Identité sociale et conflit}

Selon Tajfel, Billig, Bundy et Flament (1971), "l'identité sociale est étroitement liée à la position que le sujet occupe dans la société ». Le maintien d'une l'identité sociale positive est basé sur le processus de comparaison sociale (Festinger, 1971). Afin d'éviter toute crise identitaire, les individus vont mettre en place diverses stratégies. "Les stratégies identitaires apparaissent comme le résultat de l'élaboration individuelle et collective et elles apparaissent dans les ajustements opérés en fonction de la variation des situations et des finalités exprimées par les acteurs » (Camilleri et collab., 1990, p. 49).

Or « l'image héroïque des origines que la lutte pour la reconnaissance des identités a forgée ne permet pas [...] aux Antilles françaises de souder les sujets autour d'une mémoire nationalitaire. Les résistances, essentiellement le marronnage et la fuite hors des plantations et des habitations, ne furent pas à la hauteur des attentes d'une histoire noble, combative et victorieuse. À telle enseigne que ce déficit d'héroïsme s'est retourné en logique vindicatoire " (Affergan, 2006, p. 54). Le modèle "rejet-identification » développé par Branscombe, Schmitt et Harvey (1999) prévoit en effet que certains individus, lorsqu'ils sont rejetés ou discriminés, auraient tendance à accentuer leur identification au groupe ethnique pour augmenter leur bien-être subjectif, alors qu' « une identité culturelle reconnue et valorisée contribue au maintien d'une identité sociale positive et favorise la sécurité identitaire » (Bourhis et Bougie, 1998, p. 77).

Nous supposons dans ce contexte que le conflit va avoir un rôle dans la dynamique identitaire des groupes. Nous nous intéressons ici au conflit social car il « implique toujours une action sociale réciproque » (Coser, 1982, p. 24). Cette acception implique une altérité et une réciprocité dans l'action sociale. "Le conflit sert à établir et à maintenir l'identité et les limites des sociétés et des groupes. Le conflit avec d'autres groupes contribue à l'établissement et à la réaffirmation de l'identité du groupe et maintient ses limites par rapport au monde social qui l'entoure " (Coser, 1982, p. 23). Le conflit est appréhendé comme un moyen de maintenir une délimitation entre les groupes et permet à leurs membres de se positionner, de maintenir leur identité. Il peut être également pourvoyeur de changement, notamment quand on est face à un rapport hiérarchique. Les groupes majoritaires ont tendance à préférer le contrôle social au changement, car " leur idéal, et en réalité leur affaire, c'est l'équilibre, la résolution indolore des conflits, l'adhésion aveugle aux opinions et aux lois existantes, et à la propagation d'une seule conception de la réalité " (Moscovici, 1979, p. 108). Les minorités ont tendance à lutter contre les discriminations, quitte à enfreindre les règles établies et à renoncer à la stabilité. Cette volonté des minorités d'obtenir le changement va créer un désaccord, qui va être perçu comme créateur d'angoisse. 


\section{Conflit identitaire et sentiment de capacité}

Il y a conflit lorsqu'une décision ne peut être prise par les procédures habituelles (March et Simon, 1991/1958). Or l'identité est définie comme un processus de représentation de soi par lequel les individus organisent leur expérience (Zavalloni et Louis-Guérin, 1984). En effet, la représentation de soi est une succession d'étapes, alternant phases d'acuité représentationnelle et phases de diffusion représentationnelle. Le conflit identitaire s'explique par un déséquilibre entre la phase d'acuité représentationnelle, qui se traduit par de la clairvoyance cognitive où le sujet maîtrise son environnement et est capable d'organiser ses compétences, et la phase de diffusion représentationnelle, liée selon Costalat-Founeau (1999) à une dissonance entre capacité objective et subjective. Un individu ayant une forte estime de soi va lutter activement contre l'échec (Brown et Smart, 1991). Dans sa quête de reconnaissance, il trouvera la capacité d'entreprendre des actions qui vont certes générer du conflit, mais aussi équilibrer son système capacitaire (Costalat-Founeau, 1994).

Les mécanismes de construction identitaire passent alors par des ancrages sociaux provisoires (Costalat-Founeau, 1997; Guillen, 2005). Le conflit social qu'a connu la Martinique, par sa valeur normative, a permis à ses habitants de se construire une identité virtuelle (Dubar, 1991; Sainseaulieu, 1998) : «ils doivent faire surgir une nouvelle lisibilité de la réalité concrète et [...] deviennent parfois créateurs d'un univers nouveau dont eux-mêmes, génèrent le sens " (Vinsonneau, 2005). Ce nouvel espace de reconnaissance est susceptible de leur servir de cadre de référence, de champ de comparaison sociale.

\section{L'approche ego-écologique}

L'analyse ego-écologique (Zavalloni et Louis-Guérin, 1984), prend en considération la complexité liée au fait de considérer à la fois la notion de réalité objective et son aspect subjectif, c'est-à-dire l'univers des expériences. L'identité est alors appréhendée " comme une modalité particulière de construction de la réalité, un point de vue à partir duquel le monde extérieur devient monde intérieur en fonction d'un projet et d'une histoire » (Louis-Guérin et Zavalloni, 1987, p. 67).

Le point de départ de l'approche ego-écologique consiste à considérer toute personne comme située dans une matrice sociale formée par les différents groupes sociaux auxquels elle appartient ou desquels elle se dissocie. D'une part, ces groupes représentent des catégories sociales d'appartenance - et constituent ainsi une identité sociale objective de la personne - et, d'autre part, ils sont investis d'une dimension symbolique, émotionnellement chargée, qui est à l'origine de toute construction identitaire. Le groupe est en soi un concept abstrait et, pour être doté de sens, il doit être recodé en images particulières, souvent incarnées par des sous-groupes - et renvoyant alors à des groupes primaires comme les parents, les amis ou les collègues - ou par des personnes, réelles (proches, écrivains, artistes, etc.) ou imaginaires. Ces images déterminent le sens et la valeur de chacun des groupes d'appartenance ou de non-appartenance. En ce sens, les sous-groupes ou personnes concrètes qui soustendent ce vécu subjectif représentent des prototypes identitiels.

Le recodage du groupe est le premier des mécanismes à l'origine de la construction de l'identité dans l'approche ego-écologique. II traduit la façon dont l'individu découpe le monde dans lequel il vit et évolue en des unités dotées de sens, intégrant l'histoire personnelle et collective et des éléments liés à la connaissance, à l'affect et aux projets. Il permet de passer d'un concept abstrait à des référents empiriques (Zavalloni et Louis-Guérin, 1988). Le deuxième mécanisme est celui de la différenciation et de l'opposition binaire entre le Soi et le Non-Soi, le positif et le négatif. Ce mécanisme implique que toute identité se construit par opposition à une altérité et, inversement, par identification à autrui. Le troisième mécanisme identitaire est celui de la "réversibilité » entre le Je et Nous. En effet, les représentations du monde, de la société, du groupe et d'alter et les représentations de soi sont liées. Afin de se représenter elle-même, la personne doit se situer dans son environnement, par rapport aux autres et à la société. Le groupe ne devient concret qu'à travers les sous-groupes et les personnages prototypes qui ont été choisis comme référents privilégiés. Le sens dont autrui est porteur, aux yeux de la personne, dépend des relations, réelles et imaginaires, avec le Soi. L'équivalence entre les représentations du groupe et du Soi est d'autant plus grande que l'identification au groupe est forte (Louis-Guérin et Zavalloni, 1987). 


\section{Méthodologie}

L'Investigateur multistades de l'identité sociale (IMIS) est un outil d'objectivation, un moyen d'obtenir des données brutes qui permettent ensuite de repérer des phases identitaires et des processus (Zavalloni et Louis-Guérin, 1984). C'est une démarche exploratoire qui se déroule en trois phases. Un livret comporte différents groupes stimuli auxquels on peut soumettre un individu afin d'activer sa dynamique identitaire. Le choix des groupes se fait en fonction des objectifs de la recherche. Nous avons analysé cinq cas avant et cinq cas après le conflit social qu'a traversé la Martinique. Cependant, nous avons choisi de ne présenter les résultats que pour un seul participant, du fait de son caractère emblématique.

\section{IMIS, phase I : répertoire des représentations}

La première phase consiste à élaborer le répertoire des unités représentationnelles pour chaque sujet participant, à travers des questionnaires se rapportant chacun à un groupe social. Le sujet doit fournir, pour ces groupes stimuli, des mots ou des courtes phrases les définissant le mieux, en faisant la distinction Nous/Eux (sauf dans le cas de l'exogroupe). Cette distinction doit permettre au chercheur de déterminer l'ambiguïté des relations entre l'individu et le groupe. Nous avons choisi les groupes stimuli suivants pour cette recherche : la nationalité, le sexe, la région (appartenance territoriale), l'activité professionnelle et la spiritualité (avant le conflit).

\section{IMIS, phase II : élucidation du sens des représentations}

Dans cette phase, l'accent est mis sur le sens que les unités représentationnelles ont pour l'individu. On met en exergue le microcosme social du sujet, c'est-à-dire l'ensemble des référents implicites qui incarnent les représentations que le sujet se fait du monde. On essaie, ensuite, de découvrir les caractéristiques communes ou divergentes du sujet en lien avec les groupes d'appartenance et de non-appartenance.

- Niveau 1 : microcosme social, recodage des groupes et images prototypes. Ce premier niveau a pour objectif de faire émerger le microcosme social de la personne, c'est-à-dire les référents implicites des représentations qui déterminent la façon dont l'individu appréhende la réalité sociale. Ces référents implicites constituent les prototypes identitiels. Ils servent à découper le monde social par rapport aux références inhérentes au sujet et non à partir des catégories sociales considérées a priori.

- Niveau 2 : propriétés élémentaires des représentations : egomorphisme (Soi) et valeurs. Le deuxième niveau de l'introspection focalisée permet de déceler les traits communs et les différences de caractéristiques entre le Soi et les groupes d'appartenance ou de non-appartenance. II permet également de savoir quelle valeur et quelle importance le sujet attribue aux unités représentationnelles données.

- Niveau 3 : signification différentielle des qualités pour Soi et pour alter. La dernière étape de la deuxième phase consiste à élucider le sens que chaque représentation produite a pour la personne lorsqu'elle est appliquée au Soi et lorsqu'elle est appliquée au groupe (individuel/collectif).

\section{IMIS, phase III : déploiement de l'environnement intérieur opératoire}

Dans cette phase, le sujet fournit aux unités représentationnelles allomorphiques (Non Soi) une signification, et les unités représentationnelles egomorphiques (Soi) élucident la charge émotionnelle ainsi que le degré d'application. Cela lui permet donc de prendre conscience de son environnement intérieur opératoire au cours d'un entretien semi-directif. Cette phase correspond à l'exploration du contexte sous-jacent de chaque unite représentationnelle, ou ce que nous avons appelé la pensée de fond. En situation naturelle, ce contexte demeure dans le non-dit, à la périphérie de la conscience, mais il est immédiatement mobilisable lorsque l'on demande à une personne d'expliquer ses représentations du monde et de soi. Elle évoque alors des personnages, une histoire, des souvenirs, des sentiments et toute une axiologie qui renvoie au système de croyances et de valeurs, à la motivation et au projet de vie. De plus, l'explication des liens et des relations qui existent entre les éléments du circuit affectivoreprésentationnel nous permet d'élucider les motivations profondes du sujet par le repérage de noyaux dynamiques socio-motivationnels, qui sont des groupements thématiques de mots entre eux, puis de thèmes entre eux. Ces noyaux correspondent à un sous-système de l'identité qui relie un ensemble de mots dont le contenu 
sémantique est étroitement corrélé, indépendamment de leur position dans les cases de l'espace élémentaire. II existe différentes relations entre les mots dans la dynamique identitaire d'un individu :

- Contre élaboration défensive du Soi : une qualité personnelle (Soi+) qui permet de contrebalancer un défaut personnel (Soi-)

- Blocage : un défaut d'alter (Non Soi-) bloque l'expression d'une qualité personnelle (Soi+)

- Opposition : une qualité personnelle (Soi+) s'oppose à un défaut d'alter (Non Soi-)

- Support : une qualité d'alter (Non Soi+) soutient une qualité personnelle (Soi+) ou permet de palier un défaut personnel (Soi-)

- Contiguïté : deux caractéristiques se renforcent mutuellement.

- Implication : une caractéristique en implique une autre.

Ces liens représentent des circuits affectivo-représentationnels qui peuvent être décelés soit par le biais d'un travail interprétatif des données discursives, soit en collaboration avec le participant. On peut alors mettre en évidence les configurations passées, présentes et potentielles de l'identité du sujet, en relation avec la représentation de Soi, d'alter et de la société.

\section{Mots identitaires}

L'analyse psycho-contextuelle utilise comme point de départ les représentations du monde social et permet ensuite de générer les images et souvenirs qui les accompagnent. Une fois cet ensemble obtenu, chaque unité représentationnelle devient ce que l'on a appelé un mot identitaire, qui renvoie à un ensemble d'images, d'idées, d'affects, de valeurs et de représentations que l'individu accumule tout au long de sa vie et qui porte tout le système identitaire. Ces mots ne sont pas nombreux, mais ils reviennent incessamment dans le discours que l'individu tient sur soi, sur alter ou sur la société. Ils ont tendance à survivre tout au long de la vie, même si leur sens est susceptible de changer au fil du temps. Lors de changements identitaires, les mots identitaires ne disparaissent pas, mais on assiste à des transferts de significations et de valeurs auxquelles ils renvoient. Au niveau individuel, les mots identitaires représentent des invariances transdimensionnelles. Leur structure renvoie respectivement à différentes dimensions sociales et individuelles telles que le concept de soi, la motivation, les attitudes, les valeurs, l'autobiographie et les représentations sociales.

\section{Protocole pour l'exploration des mots identitaires}

Le protocole que l'on applique pour chaque mot identitaire consiste à interroger la personne sur :

- les connotation des unités représentationnelles egomorphes positives (Soi+)

- les connotation des unités représentationnelles egomorphes négatives (Soi-)

- les connotations des unités représentationnelles allomorphes positives (Non Soi+)

- les connotation des unités représentationnelles allomorphes négatives (Non Soi-)

- les éléments biographiques et histoire sociale liés à l'unité représentationnelle décrivant le Soi

- les éléments biographiques et histoire sociale liés à l'unité représentationnelle décrivant alter

- les éléments biographiques et histoire sociale liés à toutes les unités représentationnelles

- I'importance de l'unité représentationnelle appliquée au Soi

- l'importance de l'unité représentationnelle appliquée à alter

- les champs d'actualisation de l'unité représentationnelle appliquée au Soi

- les champs d'actualisation de l'unité représentationnelle appliquée au groupe 
- les stratégies de réalisation et le mode d'adaptation à l'environnement (exprimés par les unités représentationnelles du Soi+ et du Soi-)

- les stratégies de réalisation et le mode d'adaptation à l'environnement (exprimés par les unités représentationnelles du Non Soi+ et du Non Soi-)

- I'intégration avec le milieu: perception, évaluation et réaction du répondant face à la réaction sociale exprimée par le milieu : unités représentationnelles qui décrivent le groupe.

\section{Procédure}

Le recueil de données s'est déroulé en deux temps. Dans un premier temps, au cours d'un entretien d'environ $30 \mathrm{~min}$, le chercheur a recueilli le répertoire des unités représentationnelles du participant et de leurs spécifications (Soi et Non-Soi, valeurs positives et négatives). Ensuite a eu lieu un entretien d'explicitation, au cours duquel l'individu a recontextualisé chaque unité représentationnelle. Ce second entretien, de 3 h, a été enregistré pour obtenir une retranscription du discours et pour pouvoir procéder à une analyse des thèmes émergents. L'analyse des relations nous a permis d'élucider les motivations profondes du sujet par le repérage des noyaux dynamiques socio-motivationnels. La recherche s'est déroulée en deux temps : en juillet 2008, puis en juillet 2009. Les résultats de ces deux étapes nous ont permis d'obtenir les indices d'une société en pleine mutation et de voir apparaître divers éléments d'une dynamique identitaire à l'œuvre.

\section{Participant}

Nous avons interrogé Anthony Angel 4 fois, 2 fois avant et 2 fois après le conflit. II est né et vit en Martinique. En 2008, il a 44 ans, est marié et sans enfant. II a vécu en France métropolitaine pendant ses études universitaires. II est cadre dans le secteur public, bras droit du député maire d’une des communes de la Martinique et il nous a dit se sentir très impliqué dans le développement de l'île. Cet investissement dans la vie de l'île passe non seulement par son activité professionnelle, mais par son investissement politique, bien qu'il ne détienne aucun mandat, et par des tribunes publiées dans des magazines traitant de faits de société, et ce, notamment pendant le conflit.

\section{Résultats}

L'explication des relations qui existent entre les éléments du circuit affectivo-représentationnel du sujet nous permet d'élucider ses motivations profondes par le repérage des noyaux dynamiques socio-motivationnels. Nous présentons ici un tableau récapitulatif des relations entre les unités représentationnelles que nous avons identifiées et la dynamique identitaire du participant (tableau 1, page suivante).

\section{Avant le conflit : relation de dominant/dominé entre la France et la Martinique}

L'analyse a mis en évidence de manière générale chez les Martiniquais le thème du besoin de valorisation, illustré par les unités repréentationnelles blessé, métissé et en quête de valorisation, thème qui entre en résonance avec le thème de la supériorité, illustré par des unités représentationnelles telles que expansionniste, arrogant, non tolérant, se croient supérieurs, n'ont pas réglé les contingences post-coloniales attribuées au groupe des Français. Ces thèmes sont liés car, selon Anthony Angel, il existe une supériorité, une domination de la France sur la Martinique, ce qui fait naître un besoin de valorisation lié à la quête de reconnaissance. Selon Camilleri (1997), cela correspond à des stratégies où le sujet va chercher à valoriser son groupe et son identité (voir aussi Camilleri et Cohen-Emerique, 1989; Camilleri et collab., 1990, Camilleri et Vinsonneau, 1996). Ici, Anthony Angel distingue les Martiniquais des Français, confirmant les propos tenus lors du premier entretien : il ne se considère pas comme Français. Il y a là une distinction entre la nationalité et la citoyenneté : se sentir citoyen, c'est être reconnu comme membre à part entière de la cité. Or le besoin de valorisation et le sentiment d'être dominé laissent à penser qu'il est citoyen au sens juridique du terme, mais qu'il n'éprouve pas d'affiliation nationale (Fortin, 2001). Nous retrouvons également l'unité représentationnelle peu citoyen, qui offre une définition de la citoyenneté comme étant le ciment entre les individus. Selon Anthony Angel, les Martiniquais sont peu citoyens, et c'est un élément qu'il juge comme indésirable puisqu'il le classe dans le Non Soi-. Cela va dans le sens d'Affergan (2006), qui a constaté, chez les Martiniquais, un irrespect manifeste de tout ce qui touche au domaine public, par opposition au domaine privé, ce qui est le signe que l'île échappe à la notion de propriété. 
Tableau 1. Dynamique identitaire d'Anthony Angel avant et après le conflit

\begin{tabular}{|c|c|c|c|c|}
\hline \multirow{2}{*}{$\begin{array}{l}\text { Types de } \\
\text { relation }\end{array}$} & \multicolumn{2}{|l|}{ Avant le conflit } & \multicolumn{2}{|l|}{ Après le conflit } \\
\hline & Mots identitaires & Cotation & Mots identitaires & Cotation \\
\hline \multirow{2}{*}{$\begin{array}{l}\text { Contre- } \\
\text { élaborations } \\
\text { défensives } \\
\text { du Soi }\end{array}$} & $\begin{array}{l}\text { Multiculturel / Trop circonscrits à leur } \\
\text { sphère }\end{array}$ & S1E+/S2- & Accueillants/ Difficiles & $\mathrm{S} 1+/ \mathrm{S} 2-$ \\
\hline & $\begin{array}{l}\text { Travailleur / Faussement indépendant } \\
\text { Chaleureux / En solitude }\end{array}$ & $\begin{array}{l}\mathrm{S} 1 \mathrm{E}+/ \mathrm{S} 2- \\
\mathrm{S} 2 \mathrm{E}+/ \mathrm{S} 1-\end{array}$ & Avons une bonne image / Préoccupés & $\mathrm{S} 1 \mathrm{E}+/ \mathrm{S} 1-$ \\
\hline Blocages & Non rigoureux / Exigeant & NS1E-/S1E+ & $\begin{array}{l}\text { Métissé / N’ont pas réglé le passif colonial } \\
\text { Métissé / Raciste } \\
\text { Privilégié / Non solidaire }\end{array}$ & $\begin{array}{l}\text { S1E+/NS1E- } \\
\text { S1E+/NS2E- } \\
\text { S1E+/NS2E- }\end{array}$ \\
\hline \multirow[t]{3}{*}{ Oppositions } & $\begin{array}{l}\text { Expansionniste / N'ont pas réglés les } \\
\text { contingences post-coloniales }\end{array}$ & S2+/NS1E- & Complexé / Accueillant & NS1E-/S1E+ \\
\hline & Ambitieux /Pas assez rêveur & S1+/NS1- & Non rigoureux / Sollicités & NS1E-/S1E+ \\
\hline & $\begin{array}{l}\text { En devenir / En quête de valorisation } \\
\text { Respecté / Arrogant }\end{array}$ & $\begin{array}{l}\text { S2E+/NS1- } \\
\text { S1+/NS2- }\end{array}$ & Irresponsable / Bien payé & NS1E-/S2+ \\
\hline \multirow[t]{3}{*}{ Supports } & $\begin{array}{l}\text { Pas assez nombreux / Jouissent d'un } \\
\text { grand pays }\end{array}$ & S2-/NS2E+ & Riche / Privilégié & NS2+/S1E- \\
\hline & $\begin{array}{l}\text { Une valeur ajoutée non optimisée / } \\
\text { Jouent un rôle majeur en Europe }\end{array}$ & S1-/NS1+ & Corporatiste / Pas assez représentatif & $\mathrm{NS} 2+/ \mathrm{S} 2-$ \\
\hline & Pas toujours au clair / Courageux & S2-/NS1+ & & \\
\hline \multirow[t]{2}{*}{ Contiguïtés } & $\begin{array}{l}\text { N'ont pas réglés les contingences post } \\
\text { coloniales / En quête de valorisation }\end{array}$ & NS1E-/NS1- & Aime faire la fête / Bon vivant & $\mathrm{S} 2+/ \mathrm{S} 2+$ \\
\hline & Pas assez rêveur / Trop cartésien & NS1-/NS1- & Ont un beau pays / Privilégiés & S1E+/NS1- \\
\hline \multirow[t]{2}{*}{ Implications } & Métissé / Multiculturel & $\mathrm{S} 1 \mathrm{E}+/ \mathrm{S} 1 \mathrm{E}+$ & $\begin{array}{l}\text { Prétentieux / Se croient le centre du } \\
\text { monde }\end{array}$ & S2+/NS1- \\
\hline & Pas assez féminin / Macho & S1E-/NS1E- & $\begin{array}{l}\text { Aiment la réforme immobile / } \\
\text { Conservateur }\end{array}$ & S3-/NS1- \\
\hline
\end{tabular}

Abréviations : +, -, valeurs positive et négative; 1,2,3, niveaux d'élucidation du sens; NS, Non Soi; S, Soi.

Remarque : voir la section méthodogique pour l'explication des différents types de relation, du concept de mot identitaire et pour la signification détaillée de la cotation.

\section{Avant le conflit : référent négatif, espace de liberté et résonance interne}

Dans le discours d'Anthony Angel, une thématique est commune à la fois au groupe des hommes et au groupe des Martiniquais, ce qui fait qu'il parle de l'homme martiniquais en général. Cette thématique est celle de l'irresponsabilité, du manque de respect envers autrui et envers sa propre famille, ce qui est illustré par des unités représentationnelles telles que : festif, peu citoyen, macho, pas assez responsable, n'assument pas leur statut de modèle, faussement indépendant. On voit là s'esquisser une image du référent identitaire (en général la famille ou un membre de l'entourage plus ou moins proche) qui est négative, l'image paternelle y revêtant notamment le caractère de prototype négatif. II s'agit d'un individu qui posséderait non pas toutes les qualités et compétences auxquelles le sujet peut se référer, comme un prototype identitaire positif, mais au contraire tous les éléments rejetés, les défauts. On peut imaginer que cet élément négatif auquel le sujet se réfère lui permet de prendre le contre-pied de celui-ci, ce qui lui offre un espace de liberté quant à la création d'un prototype idéal. L'irresponsabilité et le manque de respect pour les règles établies seraient, selon de nombreux anthropologues qui ont qualifié ce phénomène de caractéristique d'une "société permissive " - la conséquence d'une violente réaction à l'enfermement de l'esclavage et d'une absence de hiérarchisation des normes (Affergan, 2006). On voit d'aialleurs apparaître une opposition entre certaines unités représentationnelles attribuées au groupe des hommes et des Martiniquais et celles attribuées au groupe des cadres. En effet, le sujet attribue au groupe des cadres des unités représentationnelles telles que privilégié, respectés, en devenir, travailleur et leur oppose des unités représentationnelles telles que en solitude, pas assez rêveurs, trop circonscrits à leur sphère. Cette opposition laisse à penser que, dans la société martiniquaise, le statut de cadre, en plus de son rôle au niveau de l'organisation, revêt une importance toute particulière au niveau de la société elle-même. Ce statut confère une responsabilité à ceux qui en sont pourvus et cette notion de responsabilité fait écho à l'absence de l'homme au sein de la cellule familiale dans la société martiniquaise. Il lui permet de s'inscrire dans une autre dynamique, celle de l'homme capable, responsable, valorisé. Ce statut lui offre un espace de liberté dans cette identité en pleine mutation et la 
possibilité d'accéder à la reconnaissance. De plus, on retrouve un nombre important d'unités représentationnelles dans le Non Soi+, qui correspond aux aspirations, à la différenciation positive, à la sphère du projet. Les éléments du Non Soi+ sont des caractéristiques et des compétences valorisées, voir enviées par l'individu et qu'il attribue à un prototype identitaire, l'individu par excellence, le modèle, l'opposé de tout ce que l'on retrouve dans le Non Soi-. L'IMIS permet de mettre en évidence des frustrations, des victimisations, des aspirations et des qualités que l'individu partage avec ses groupes d'appartenance ou de non-appartenance, et qui sont des éléments essentiels à la compréhension du processus de représentation de soi.

\section{Après le conflit : thème de l'irresponsabilité}

L'analyse des unités représentationnelles du groupe des Martiniquais et du groupe des hommes nous a permis de mettre en évidence un thème commun, celui de l'Irresponsabilité, illustré par des unités représentationnelles telles que irresponsable, non solidaire, macho et lâche pour le groupe des hommes et irresponsable, difficile et non rigoureux pour le groupe des Martiniquais. Cette thématique traverse les deux groupes, le sujet faisant référence à I'Irresponsabilité, car « les Martiniquais ont des attitudes à travers lesquelles ils s'affranchissent de leur devoir de citoyen", mais également au côté lâche des hommes, car "ils n'assument pas toujours leurs actes ou les conséquences d'éléments extérieurs ». Le sujet illustre ses propos concernant cette thématique par des exemples concernant les rapports hommes/femmes, explicitant l'unité représentationnelle macho (" c'est cultiver un rapport inégalitaire avec la femme, un rapport qui lui est défavorable ") et faisant référence à la place du père dans la société martiniquaise et à l'inégalité dans la répartition des tâches domestiques. Chez le sujet, ce thème apparaît pour les hommes martiniquais en tant qu'individus mais aussi en tant que citoyens.

\section{Après le conflit : thème de l'immobilisme}

L'unité représentationnelle métissé est définie ainsi: "cela comporte deux aspects, l'un ethnique et l'autre intellectuel, culturel », elle est illustrée par la composition ethnique, par la diversité des pratiques culturelles et culinaires, par rapport à la musique et elle est cotée comme étant extrêmement positive. On retrouve également l'unité représentationnelle complexé qui est attribuée aux Martiniquais et qui est définie comme suit: "nous n'avons pas réglé le contentieux, le passif de l'esclavage et on ne nous a pas aidé à le régler, c'est partagé. Nous sommes sur la défensive, nous percevons les choses au travers du regard extérieur, celui de la France hexagonale ». Le sujet illustre ses propos par une expression créole, "la po sauvé ", ce qui signifie être clair de peau. II se réfère au fait qu'en Martinique les gens font une gradation dans la couleur de la peau, ce qui participe à l'entretien du complexe. Ce complexe est lié à la signification des unités représentationnelles telles que n'ont pas réglé le passif colonial, conservateur et se croient le centre $d u$ monde et que nous regroupons sous la thématique de l'immobilisme. En effet, que les Martiniquais soit un peuple métissé mais complexé se justifie pour le sujet par le fait que la France n'ai pas réglé le passif colonial. Il explique ce complexe par une absence de reconnaissance de l'identité, de l'histoire martiniquaise qui est liée à celle de la France par la colonisation. Anthony Angel confirme sa position concernant l'immobilisme de la France et le fait qu'elle n'ait pas soldé son passé colonial en faisant référence aux lois de 2005 sur les effets positifs de la colonisation : " cela traduit un état d'esprit, une manière de penser, une supériorité de la race, de la culture franco-française sur le reste; cela participe d'une certaine forme d'asphyxie de la différence, on ne solde pas les comptes de l'histoire; cela génère des tensions et des pathologies collectives ". L'immobilisme de la France quant à la reconnaissance d'une identité martiniquaise et d'une histoire commune (symbolisée par exemple par la récente reconnaissance par l'État français des vétérans martiniquais, des dissidents ayant participé à la Seconde Guerre mondiale, plus de 60 ans après les faits) créerait un complexe qui se manifesterait dans l'affrontement. En effet, « l'ancien conflit entre le Maître et l'Esclave [...] s'est métamorphosé en une dissension endogène, l'Antillais d'aujourd'hui devant, pour prendre conscience de sa propre identité, lutter contre le Français qu'il a voulu lui-même devenir " (Affergan, 2006, p. 12). Le statu quo dans la relation entre la France et la Martinique viendrait du fait que, pour affirmer son identité, le Martiniquais doit lutter contre le Français qui est le symbole d'un passé non digéré et intériorisé.

\section{Après le conflit : thème du privilège}

Chez Anthony Angel, la thématique du privilège traverse tous les groupes d'appartenance, et elle est parfois connotée positivement, notamment quand il dit : "Nous les Français, nous sommes... ", "Nous les cadres, nous 
sommes [...] " et négativement en ce qui concerne les autres groupes d'appartenance, comme " Eux les cadres, ils sont [...] ». L'unité représentationnelle privilégié, quand elle est attribuée au groupe des Français, est à associée à riche et met en avant l'aspect économique, "le système de protection sociale qui fait envie [...] ", et le sujet fait d'ailleurs référence aux patrons du CAC 40. Cette thématique du privilège, quand elle est connotée positivement pour le groupe des Français, est également illustrée par des unités représentationnelles telles que ont un grand et beau pays, ont une bonne image et prétentieux et, pour le groupe des cadres, on retrouve des unités représentationnelles telles que enviés (apparaît deux fois) et bien payés. En effet, ils sont enviés " par des gens de conditions inférieures [...] », bien payés " c'est-à-dire avoir un niveau de rémunération au-dessus de la moyenne ». L'unité représentationnelle privilégié, lorsqu'elle est connotée négativement, est à mettre en lien avec « Eux les cadres [...] » avec l'unité représentationnelle pas assez représentatif. En effet, le sujet définit privilégié comme : "Les cadres ne sont pas dans la même galère que les autres, tant au niveau économique qu'au niveau de l'épanouissement » et ils forment un population pas assez representative : " [...] la population des cadres devrait être à l'image de la population, en termes de démocratisation de l'accès à l'encadrement [...] ». Quand Anthony Angel attribue cette unité représentationnelle privilégié au groupe des Martiniquais, il fait référence à « un système économique et social hors norme qui est au-dessus de nos capacités de production, rapporté à notre environnement immédiat » et, pour le groupe des hommes, il fait référence au rapport déséquilibré entre hommes et femmes avec l'unité représentationnelle plus instruit, qui est également connotée négativement. Le sujet distingue le versant positif du versant négatif de privilégié au niveau des répercussions et de la répartition des privilèges, qu'il juge plus ou moins égalitaire ou justifiée en fonction du contexte. Ce thème du privilège est également un moyen pour le sujet de faire valoir une image de soi positive, socialement valorisée, qu'il entend voir confirmer (par le groupe qu'il rejette.

\section{Discussion}

Les résultats obtenus montrent que la quête de reconnaissance pourrait mener au conflit, influer sur le sentiment d'appartenance et affecter la dynamique identitaire.

Le sujet ne se sent pas français. Nous le percevons comme un rejet, et nous nous sommes donc interrogés sur les causes de ce rejet. Selon Goffman (1973), lors de toute interaction, l'image de chaque individu est exposée à l'autre, pour défendre son propre territoire (propriétés de l'individu et de l'ensemble de ses prolongements corporels, matériels, spatiaux et affectifs), puis pour proposer une image de soi valorisante (élaboration et projection de l'image de soi dans l'interaction). Anthony Angel tenterait donc, par ce rejet du groupe des Français, de présenter une image positive socialement valorisée de lui-même. La Martinique, ancienne colonie française, est devenue un département d'outre-mer (DOM), donc il existera toujours un rapport de position déséquilibré (Picard, 2008) avec la France, d'où la nécessité pour l'individu de protéger son territoire et de proposer une image valorisante de lui-même. Les Martiniquais mettraient ainsi en place des stratégies de revalorisation de leur singularité (Camilleri et Cohen-Emerique, 1989) qui contribueraient à la conservation d'une bonne image de leur groupe (Tajfel et Turner, 1979), et ceci se ferait au détriment de celui des Français.

Le sujet a attribué des stéréotypes au groupe des Français notamment pour le " Nous ", nous nous sommes donc interrogés sur l'utilisation de ceux-ci lors du recodage. Selon Tajfel et Turner (1979), les individus peuvent être considérés comme faisant partie d'un groupe d'appartenance s'ils se définissent comme faisant partie de celui-ci et si les autres les définissent comme tels. Le fait que le sujet ne reconnaisse pas les Français comme groupe d'appartenance serait lié au fait qu'il ne se définisse pas comme tel, mais également au fait qu'il ne serait pas défini comme tel par les autres. Cela expliquerait l'utilisation de stéréotypes, car « un stéréotype est l'ensemble des croyances d'un individu relatives aux caractéristiques ou aux attributs d'un groupe " (Judd et Park, 1993). Nous avons suivi également une autre piste, celle de la simplexité. Berthoz (2009) considère la simplexité comme « [...] l'ensemble des solutions trouvées par des organismes vivants pour que, malgré la complexité des processus naturels, le cerveau puisse préparer l'acte et en projeter les conséquences. Ces solutions sont des principes simplificateurs qui permettent de traiter des informations ou des situations en tenant compte de l'expérience passée et en anticipant l'avenir ». Cette simplification pourrait être une autre explication quant au choix qu'a fait le sujet d'utiliser des stéréotypes quand il s'agit des Français. Les Martiniquais sont culturellement différents des Français et ne partagent pas la même histoire, à l'exception de quelques événements douloureux. Le choix fait par 
Anthony Angel concernant les stéréotypes relèverait de la simplexité, c'est-à-dire d'un moyen simple de traiter la complexité, complexité dans laquelle nous l'avons placé en imposant le groupe " nationalité » lors des entretiens. Nous soulignons également la fonction facilitatrice des stéréotypes dans la description du groupe. Selon SalesWuillemin (2005), au cours d'une situation où les appartenances groupales sont mobilisées, les stéréotypes intériorisés par les individus peuvent être rapidement mobilisés. L'utilisation de ces stéréotypes est un moyen d'évacuer des frustrations. Ce besoin d'évacuer des frustrations, cette quête de reconnaissance, serait la résultante d'un rapport de position déséquilibré (Picard, 2008) entre la France et la Martinique, qui s'expliquerait non seulement par le statut d'ancienne colonie, mais aussi par l'hégémonie exercée par les békés sur l'économie. De plus, ce déséquilibre est à l'origine d'un besoin pour les Martiniquais de considération de leurs particularités et, de ce besoin, naît un conflit tant au niveau identitaire que social, qui les amène à libérer des capacités d'action. «En effet, dans la mesure où l'expérience de la reconnaissance sociale est une condition dont dépend le développement de l'identité personnelle dans son ensemble, l'absence de cette reconnaissance, autrement dit le mépris s'accompagne nécessairement du sentiment d'être menacé de perdre sa personnalité " (Honneth, 2008, p. 193).

\section{Conclusion}

Notre recherche avait pour but de souligner les particularités de la dynamique identitaire des Martiniquais représentés ici par un cas emblématique -, qui appartiennent à une société complexe du fait de son statut, de son histoire, de ses revendications et de la cohabitation sur un même territoire de différentes cultures. Nous avons tenté de mettre en évidence ces particularités grâce à l'approche de l'égo-écologie et à l'IMIS. Nous avons ainsi pu observer l'utilisation de stéréotypes et un rejet du groupe des Français, ainsi que la mise en place de stratégies de reconnaissance. Nous avons vu que la quête de reconnaissance s'est révélée génératrice de conflit et régulatrice de l'identité.

Le sujet dont nous avons analysé les propos a tendance à utiliser des stéréotypes lorsqu'il s'agit de décrire les Français. Nous proposons deux explications à ce phénomène. Selon la première, le cas étudié ferait appel à une forme de stratégie afin de manifester son besoin de reconnaissance, en rejetant le groupe des Français et en utilisant des stéréotypes pour le décrire. Ces stratégies sont de type ontologique, c'est-à-dire qu'elles visent à valoriser l'identité de l'individu, le sens et les valeurs du groupe (Camilleri, 1997). La seconde proposition se base sur la théorie de simplexité (Berthoz, 2009), selon laquelle les solutions les plus simples sont utilisées afin de traiter de la complexité. De plus, nous pensons que l'utilisation de stéréotypes revêt une fonction facilitatrice dans la description du groupe et qu'elle est également un moyen d'évacuer des frustrations par rapport au groupe dominant (Sales-Wuillemin, 2005).

L'autre point que révèle cette recherche est le rejet du groupe des Français, que nous avons tenté d'appréhender par le biais de la " théorie de la face » de Goffman (1973) et par la mise en place d'une stratégie de revalorisation de la singularité qui a pour objectif de conserver les liens et la culture d'origine. Nous avons mis en évidence les thématiques de l'irresponsabilité, du privilège et de la fierté qui se retrouvent aux deux temps de notre recherche, et qui sont attribuées par notre participant aux groupes des hommes et à celui des Martiniquais. La thématique de la fierté met en évidence le métissage, le multiculturalisme et la fierté par rapport à un certain mode de vie. Le besoin de reconnaissance semble être ici un régulateur identitaire. En étudiant les relations entre éléments de la dynamique identitaire, nous observons en effet la présence d'éléments qui incitent à penser que le sujet est prêt à l'action, en lien avec son besoin de reconnaissance, besoin présent avant le conflit et qui ne semble toujours pas satisfait plusieurs mois après le conflit. En effet, selon nous, le sujet dont nous avons présenté ici la dynamique identitaire se trouve en phase d'acuité représentationnelle, qui se traduit comme nous l'avons vu par une clairvoyance cognitive (le sujet maîtrise son environnement et est capable d'organiser ses compétences) (CostalatFouneau, 1997). La présence de contre-élaborations défensives du soi après le conflit est le signe que les solutions apportées n'ont pas résolu le problème : le besoin de reconnaissance est toujours présent. La présence de blocages et d'oppositions sont le signe d'une certaine tension, dont l'apaisement nécessite la genèse d'un projet, une préparation à l'action. 
L'histoire des individus se déroule avec tout au long de la vie des phases plus ou moins chaotiques, où la recherche de cohérence est fondamentale et conduit à une stabilité de la représentation de soi, d'autrui et du monde. Les conflits semblent pouvoir entraîner des ruptures de cette cohérence interne et externe et générer d'autres représentations de soi, d'alter et du monde et agir sur la charge émotionnelle des mots identitaires. Ces derniers se transformeraient alors en capturant un nouveau vécu expérientiel, celui du conflit, qui enrichirait la construction identitaire.

\section{Références bibliographiques}

Affergan, F. (2006). Martinique. Les identités remarquables. Anthropologie d'un terrain revisité. Paris : Presses universitaires de France.

Benoist, J. (1972). L'Archipel inachevé. Culture et société aux Antilles françaises. Montréal : Presses de l'Université de Montréal.

Berry, J. (1976). Human ecology and cognitive style: comparative studies in cultural and psychological adaptation. Londres : Sage.

Berry, J. (1977). Introduction. Journal of Cross Cultural Psychology, 8, 131-133.

Berthoz, A. (2009). La simplexité. Paris : Odile Jacob.

Bourhis, R. et Bougie, E. (1998). Le modèle d'acculturation interactif : une étude exploratoire. Revue québécoise de psychologie, 19(3), 75-114.

Branscombe, N., Schmitt, M. et Harvey, R. (1999). Perceiving pervasive discrimination among African-Americans: Implications for group identification and wellbeing. Journal of Personality and Social Psychology, 77, 135149.

Brown, J. et Smart, S. (1991). The self and social conduct: Linking self-representations to prosocial behavior. Journal of Personality and Social Psychology, 60, 368-375.

Bruner, J. (1991). Car la culture donne forme à l'esprit. De la révolution cognitive à la psychologie culturelle. Paris : Eshel.

Camilleri, C. (1997). Les stratégies identitaires des immigrés. Sciences Humaines, 15, 32-34. (hors-série : Identité, identités : L'individu, le groupe, la société)

Camilleri, C. et Cohen-Emerique, M. (1989). Chocs de cultures : Concepts et enjeux pratiques de l'interculturel. Paris : L'Harmattan.

Camilleri, C., Kastersztein, J., Lipiansky, E., Malewska-Peyre, H., Taboada-Leonetti, I. et Vasquez, A. (dir.) (1990). Stratégies identitaires. Paris : Presses universitaires de France.

Camilleri, C. et Vinsonneau, G. (1996). Psychologie et culture : concepts et méthodes. Paris : Armand Colin.

Césaire, A. (2008). Discours sur le colonialisme suivi de Discours sur la Négritude. Paris : Présence Africaine. (1 $1^{\text {re }}$ éd. 1955)

Coser, L. (1982). Les fonctions du conflit social. Paris : Presses universitaires de France.

Costalat-Founeau, A.-M. (1994). La dynamique représentationnelle de soi, Bulletin de psychologie, 67(417), 618622.

Costalat-Founeau, A.-M. (1997). Identité sociale et dynamique représentationnelle. Rennes : Presses de l'Université de Rennes.

Costalat-Founeau, A.-M. (1999). Identity dynamic, action and context. Journal for the Theory of Social Behavior, 29(3), 289-300.

De Gaulejac, V. et Taboada-Leonetti, I. (1994). La lutte des places. Paris : Desclée de Brouwer. 
Desplan, F. (2010). Entre espérance et désespérance. Pour enfin comprendre les Antilles. Paris : Empreintes temps présent.

Doytcheva, M. (2005). Le multiculturalisme. Paris : La Découverte.

Dubar, C. (1991). La socialisation : construction des identités sociales et professionnelles. Paris : Armand Colin.

Festinger, L. (1971). Théories des processus de comparaison sociale. Dans S. Moscovici (dir.), Psychologie sociale théorique et expérimentale (p. 77-104). Paris : Mouton.

Fortin, S. (2001). Citoyennetés et appartenances en situation de migration. Les Cahiers du GRES, 2(1), 73-84.

Goffman, E. (1973). La mise en scène de la vie quotidienne. Paris : Minuit.

Guillen, S. (2005). Identité et sentiment capacitaire professionnel : une étude réalisée auprès des infirmières. Dans A.-M. Costalat-Founeau (dir.), Identité sociale et ego-écologie : théorie et pratique (p. 151-177). Paris : SIDES.

Gurrey, B. et Hokin, B. (2009). Békés, une affaire d’héritage. Le Monde [en ligne], 28 février (mis à jour le 5 mars 2009). http://www.lemonde.fr/societe/article/2009/02/28/bekes-une-affaire-dheritage_1161662_3224.html

Honneth, A. (2000). La lutte pour la reconnaissance. Paris : Cerf.

Honneth, A. (2008). La société du mépris. Vers une nouvelle théorie critique. Paris : La Découverte.

Jodelet, D. (2005). Formes et figures de l'altérité. Dans M. Sanchez-Mazas et L. Licata (dir.), L'Autre : regards psychosociaux (p. 23-47). Grenoble : Presses de l'Université de Grenoble.

Judd, C., et Park, B. (1993). The definition and assessment of accuracy in social stereotypes. Psychological Review, $100,109-128$.

Kozakaï, T. (2000). L'étranger, l'identité. Essai sur l'intégration culturelle. Paris : Payot et Rivages.

Louis-Guérin, C. et Zavalloni, M. (1987). L'ego-écologie comme étude de l’interaction symbolique et imaginaire de soi et des autres. Sociologie et sociétés, 19(2), 65-75.

Marc, E. (2005). Psychologie de l'identité. Soi et le groupe. Paris : Dunod.

March, J., et Simon, H. (1991). Organisations (J.-C. Rouchy et G. Prunier, trad.). Paris : Dunod. Ouvrage original paru en 1958 sous le titre Organizations, New York : John Wiley.

Mead, H. (1963). L'Esprit, le Soi et la Société. Paris : Presses universitaires de France.

Misantrope, Y. (2011). Identité martiniquaise et dynamique du conflit (thèse de doctorat). Université Paul Valéry Montpellier III, France.

Moscovici, S. (1979). Psychologie des minorités actives. Paris : Presses universitaires de France.

Picard, D. (2008). Quête identitaire et conflits interpersonnels. Connexions, 89(1), 75-90.

Redfield, R., Linton, R. et Herskovits, M. (1936). Memorandum for the study of acculturation. American Anthropologist, 38(1), 149-152.

Sainseaulieu, R. (1998). L'identité au travail d'hier à aujourd'hui. L'orientation scolaire et professionnelle, $27(1), 77-93$.

Sales-Wuillemin, E. (2005). Psychologie sociale expérimentale de l'usage du langage, représentations sociales, catégorisation et attitudes : perspectives nouvelles. Paris : L'Harmattan.

Sméralda, J. (2008). La société martiniquaise entre ethnicité et citoyenneté. Paris : L'Harmattan.

Taboada-Leonetti, I. (1990). Stratégies identitaires et minorités : le point de vue du sociologue. Dans C. Camilleri, E. Kastersztein, I. Lipianski, I. Taboada-Leonetti et A. Vasquez (dir.), Stratégies identitaires (p. 43-83). Paris : Presses universitaires de France. 
Taboada-Leonetti, I. (2000). Anne-Marie Thiesse - La création des identités nationales [note de lecture]. Cahier de I'Urmis, 6 [en ligne]. http://urmis.revues.org/304

Tajfel, H., Billig, M., Bundy, R. et Flament, C. (1971). Social categorization and intergroup behaviour. European Journal of Social Psychology, 1(2), 149-178.

Tajfel, H. et Turner, J.-C. (1979). An integrative theory of intergroup conflict. Dans W. Austin et S. Worchel (dir.), The social psychology of intergroup relations (p. 33-48). Monterey : Books/Cole.

Thiesse, A,-M. (1999). La création des identités nationales. Paris : Le Seuil.

Vinsonneau, G. (2005). Contextes pluriculturels et identité. Fontenay-sous-Bois : SIDES.

Zavalloni, M., et Louis-Guérin, C. (1984). Identité sociale et conscience. Introduction à l'ego-écologie. Montréal : Presses de l’Université de Montréal.

Zavalloni, M., et Louis-Guérin, C. (1988). La transdimensionnalité des mots identitaires : explorations egoécologiques. Revue internationale de psychologie sociale, 1, 173-187. 